\title{
THE LIQUID FILM FLOW WITH EVAPORATION: NUMERICAL MODELLING
}

\author{
Ekaterina Rezanova ${ }^{1,2, *}$ \\ ${ }^{1}$ Altai State University, 656049 Barnaul, Russia, \\ ${ }^{2}$ Kutateladze Institute of Thermophysics SB RAS, 630090 Novosibirsk, Russia
}

\begin{abstract}
The flow of thin liquid layer on an inclined substrate is investigated numerically. The mathematical modelling is based on the Oberbeck-Boussinesq equations and the generalized conditions on the thermocapillary boundary simplified during the parametrical analysis. In the framework of the long-wave approximation the evolution equation which determines the thickness of the liquid layer in the case of moderate Reynolds numbers is derived. The results of numerical modelling of the liquid flow with evaporation at the interface are obtained.
\end{abstract}

\section{Introduction}

A large number of both theoretical [1-5] and experimental investigations [6] are devoted to the study of viscous incompressible fluids with evaporation. The interest to such currents is caused, in particular, by the wide possibilities of their practical application in modern industrial processes. Great attention is paid to the thin liquid films. The mathematical models of the flows in the thin layer approximation and their numerical study are presented in [1-5, 7-10]. Special attention while modelling of the flows with interfaces should be given to the formulation of the boundary conditions [11-13].

In this paper we study the flow of a thin layer of viscous incompressible liquid with evaporation on the thermocapillary boundary. The motion of the liquid is accompanied by a co-current gas flow. The mathematical modelling is based on the long-wave approximation of the Oberbeck-Boussinesq convection equations and the generalized kinematic, dynamic and energetic conditions at the interface [11-13]. The evolution equation, determining the liquid layer thickness, is obtained when the effects of gravity, evaporation, capillarity, thermocapillarity and additional tangential stresses from the gas medium are taken into account (see [7-9]).

\footnotetext{
* Corresponding author: katerezanova@mail.ru
} 


\section{The problem statement. The evolution equation of the liquid layer thickness}

The thin liquid layer flow on an inclined, non-uniformly heated solid substrate in a twodimensional case is investigated. Let the coordinate system be chosen so that the $x$-axis coincides with the direction of the substrate (see fig. 1). The position of the thermocapillary interface is determined by the equation $z=h(x, t)$, the solid plate is given by the equation $z$ $=0$.

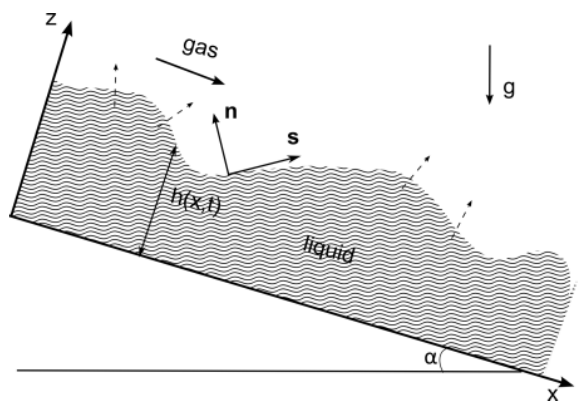

Fig. 1. Geometry of flow domain.

During the mathematical modeling of the investigated process two characteristic length scales are introduced: the transverse $-d$ and the longitudinal $-l$, the ratio of the first to the second is the small parameter of the problem $\varepsilon(\varepsilon=d / l)$. The one-sided model is based on the Oberbeck-Boussinesq equations [9]. The generalized kinematic, dynamic and energetic conditions at the thermocapillary interface after the parametric analysis in dimensionless form can be written as follows:

$$
\begin{gathered}
-\varepsilon\left(h_{t}+h_{x}-w\right)=J_{e v} E \\
u_{z}=\alpha_{\tau} \tau(x, t)-\alpha_{M a}\left(T_{x}+h_{x} T_{z}\right), \quad p=p^{g}-\alpha_{C a} h_{x x}\left(1-\alpha_{\sigma} T\right), \\
-T_{z}+\bar{\beta}_{2}\left\{\operatorname{Tdiv}_{\Gamma} \mathbf{v}\right\}=\bar{\beta}_{3} J_{e v}+\bar{\beta}_{6} h_{x x} J_{e v} .
\end{gathered}
$$

Here $\mathbf{v}=(u, w)$ is the velocity vector; $p$ is the pressure; $T$ is the temperature; $J_{e v}$ is the local mass flux at the thermocapillary interface, $E=\kappa T_{*} /\left(\lambda_{U} \rho v\right)$ is the evaporation coefficient, $\kappa$ is the heat conductivity coefficient, $\lambda_{U}$ is the latent heat of vaporization, $\rho$ is some relative value of liquid density, $v$ is the kinematic viscosity coefficient, $T_{*}$ is the characteristic temperature drop; $\alpha_{\sigma}=M a C a / P r, \quad \alpha_{M a}=\operatorname{Ma\varepsilon } / P r, \quad \alpha_{C a}=\varepsilon / C a, \quad \alpha_{\tau}=\bar{\rho} \overline{v v} \varepsilon / \bar{h}$, where $M a=\sigma_{T} T * l /(\rho v \chi)$ is the Marangoni number, $C a=u * \rho v / \sigma_{0}$ is the capillary number, $P r=v / \chi$ is the Prandtl number, $\chi$ is the thermal diffusivity coefficient, $\sigma_{T}$ is the temperature coefficient of surface tension, $\sigma_{0}$ is the value of the coefficient of surface tension $\sigma$ at the same temperature $\left(\sigma=\sigma_{0^{-}} \sigma_{T}\left(T-T_{0}\right)\right), \tau(x, t)=\left.\left(u_{z}^{g}+w_{x}^{g}\right)\right|_{\Gamma}, \bar{\rho}, \bar{v}$ are the ratio of the densities and coefficients of kinematic viscosity of the gas and liquid, $\bar{v}=u_{*}^{g} / u_{*}, \bar{h}$ is the ratio of the characteristic scale of gas layer to $l, u_{*}$ is the characteristic longitudinal velocity of the liquid; $\quad \bar{\beta}_{2}=M a \varepsilon /(\operatorname{RePrE} \bar{U}), \quad \bar{\beta}_{3}=\varepsilon, \quad \bar{\beta}_{6}=(1-1 / \bar{\rho}) \varepsilon /(\bar{U} C a), \quad \bar{U}=\lambda_{U} / u_{*}^{2} . \quad$ The dependence of the local mass flux on the temperature is determined with the help of the Hertz-Knudsen equation [3, 7-10, 13]. The no-slip conditions on the solid substrate are fulfilled and the temperature distribution $\Theta_{0}$ is set. The Reynolds number $(\operatorname{Re}=u * l / v)$ is assumed to be unity.

The problem of calculation of the unknown functions (that are the components of the velocity vector $u$ and $w$, temperature $T$ and pressure $p$ ) is studied in the thin layer 
approximation. The thickness of the liquid layer should be found for the determination of these functions. Confining to the main members of the expansions of functions $u, w, T, p$ in powers of the small parameter $\varepsilon$, and using the kinematic condition on the thermocapillary boundary, we get the evolution equation that specifies the position of the interface $h$ :

$$
\begin{gathered}
h_{t}+h_{x}\left\{\frac{h^{2}}{2}\left(\left(C_{0}\right)_{x}+\gamma_{1} \sin \alpha \Theta_{0}\right)+h C_{1}+\frac{h^{4}}{24} \gamma_{2} \cos \alpha A_{x}+\frac{h^{3}}{6}\left(\gamma_{2} \cos \alpha\left(\Theta_{0}\right)_{x}+\gamma_{1} \sin \alpha A\right)\right\}+ \\
+\left\{\frac{h^{3}}{6}\left(C_{0}\right)_{x x}+\frac{h^{2}}{2}\left(C_{1}\right)_{x}+\frac{h^{5}}{120} \gamma_{2} \cos \alpha A_{x x}+\frac{h^{4}}{24}\left(\gamma_{2} \cos \alpha\left(\Theta_{0}\right)_{x x}+\gamma_{1} \sin \alpha A_{x}\right)+\right. \\
\left.+\frac{h^{3}}{6}\left(\Theta_{0}\right)_{x} \gamma_{1} \sin \alpha\right\}+\frac{E \alpha_{J}}{\varepsilon}\left\{A h+\Theta_{0}\right\}=0 .
\end{gathered}
$$

Here $\gamma_{1}=G r / \varepsilon ; \gamma_{2}=G r ; G r=d^{3} g \beta T * / v^{2}$ is the Grashof number, $\beta$ is the thermal expansion coefficient, $\quad \alpha_{J}=\alpha_{A} \rho_{s} \lambda_{U} T_{*} / J_{*}\left(M /\left(2 \pi R_{g} T_{s}^{3}\right)\right)^{1 / 2}, \quad \alpha_{A}$ is the accommodation coefficient, $\rho_{s}$ is the vapor density, $J_{*}$ is the characteristic value of the vapor mass flux, $M$ is the molecular weight, $R_{g}$ is the universal gas constant, $T_{s}$ is the saturated vapor temperature. Functions $A, C_{0}$ and $C_{l}$ are determined by the following relations:

$$
\begin{gathered}
A=\frac{\left(-\bar{\beta}_{2}\left(C_{1}\right)_{x} h+\bar{\beta}_{3} \alpha_{J}+\bar{\beta}_{6} h_{x x} \alpha_{J}\right) \Theta_{0}}{1+\bar{\beta}_{2}\left(C_{1}\right)_{x}-\bar{\beta}_{3} \alpha_{J} h-\bar{\beta}_{6} h_{x x} \alpha_{J} h}, \\
C_{0}(x, t)=p^{g}-\alpha_{C a} h_{x x}\left(1-\alpha_{\sigma} \Theta^{0}\right)-\gamma_{2} \cos \alpha\left(A \frac{h^{2}}{2}+\Theta_{0} h\right)-\frac{\gamma_{1}}{B u} x \sin \alpha+\gamma_{2} h \cos \alpha, \\
C_{1}(x, t)=\alpha_{\tau} \tau(x, t)-\alpha_{M a} \tilde{\Theta}-\left(C_{0}\right)_{x} h-\gamma_{1} h \sin \alpha\left(A \frac{h^{2}}{2}+\Theta_{0} h\right)-\gamma_{2} \cos \alpha\left(A_{x} \frac{h^{3}}{6}+\left(\Theta_{0}\right)_{x} \frac{h}{2}\right),
\end{gathered}
$$

where $\tilde{\Theta}=A_{x} h+\left(\Theta_{0}\right)_{x}+h_{x} A, \Theta^{0}=A h+\Theta_{0}$.

The implicit finite-difference scheme, where the finite-difference analogs of the secondorder approximation are used for all derivatives with respect to $x$, for the numerical solution of the equation (2) is constructed. The problem is supplemented by periodic conditions at the ends of the studied area $x=L, x=-L$ and the initial position of the interface [10].

\section{The results of numerical investigation}

The results of numerical modelling of the thin layer flow of the liquid type as ethanol with consideration of the evaporation process in the conditions of normal gravity $\left(g=9.81 \cdot 10^{-2}\right.$ $\mathrm{m} / \mathrm{s}^{2}$ ) are presented. The initial position of the thermocapillary boundary is given by the equation $h_{0}=1-\delta_{1} \cos (k x)\left(\delta_{1}=0.01, k=\pi / 2\right)$, heating of the solid substrate is carried out according to the law $\Theta_{0}=1+\delta_{0} \cos k_{1} x \cdot \cos k_{2} t\left(\delta_{0}=0.25, k_{1}=\pi / 2, k_{2}=2\right)$.

Figure 2 shows the influence of the effects, taken into account in the study of the layer dynamics, on the boundary position. The angle of the heated substrate inclination relative to the horizon $\alpha$ is $\pi / 6$ (see figure 1 ). The coefficient $\bar{\beta}_{6}$ is assumed to be zero in the studied 
case (lines 1 and 3 of the figure 2). In cases when $\bar{\beta}_{3} \neq 0$ (lines 1 and 3 of the figure 2 ) the heat loss on the deformation of the interface as the result of the liquid evaporation are taken into account. If the term in the energy condition with a coefficient $\bar{\beta}_{2}$ is assumed unequal to zero than the consumption of energy required to overcome the surface deformation by the thermocapillary forces along the surface is taken into account (see lines 2 and 3 of the figure 2) [13]. Note that the most intense evaporation of the layer is observed when both the considered effects are assumed.

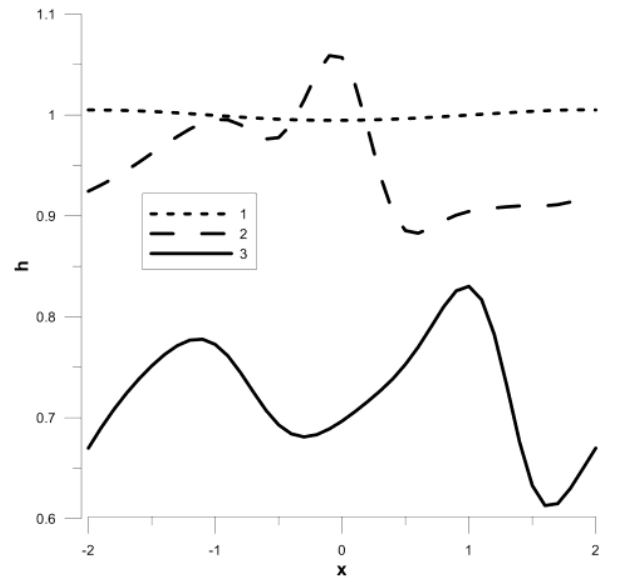

Fig. 2. The influence of various effects on the liquid layer thickness at the time $t=10^{-3}: 1$. - the position of the boundary when $\bar{\beta}_{3}=0.1, \bar{\beta}_{2}=0 ; 2$. - the position of the boundary when $\bar{\beta}_{2}=0.3$, $\bar{\beta}_{3}=0 ; 3$. - the position of the boundary when $\bar{\beta}_{2}=0.3 \bar{\beta}_{3}=0.1$.

\section{Acknowledgments}

We gratefully acknowledge the support from the Ministry of Education and Science of Russia (Agreement No. 14.613.21.0011, project identifier RFMEFI61314X0011).

\section{References}

1. A. Oron, S.H. Davis, S.G. Bankoff, Rev. Mod. Phys. 69 (3) (1997)

2. J. Klentzman, V.S. Ajaev, Phys. Fluids 21, (2009)

3. S. Miladinova, S. Slavtchev, G. Lebon, J.-C. Legros, Proc. 6th Workshop on Transport Phenomena in Two-Phase Flow, Bourgas (2001)

4. Yu.O. Kabova, V.V. Kuznetsov, O.A. Kabov, T. Gambaryan-Roisman, P. Stephan, Int. J. Heat Mass Transfer 68 (2014)

5. O. Shklyaev, E. Fried, J. Fluid Mech., 584 (2007)

6. Yu.V. Lyulin, O.A. Kabov, Int. J. Heat Mass Transfer 70 (2014)

7. O.N. Goncharova, E.V. Rezanova, Ya.A. Tarasov, Izvestia AltGU 81 (1/1), (2014)

8. O.N. Goncharova, E.V. Rezanova, Izvestia AltGU 81 (1/2), (2014)

9. O.N. Goncharova, E.V. Rezanova, Izvestia AltGU 85 (1/1), (2015)

10. E.V. Rezanova, Izvestia AltGU 89 (1), (2016)

11. C.S. Iorio, O.N. Goncharova, O.A. Kabov, Microgravity Sci. Technol 21 (1) (2009)

12. C.S. Iorio, O.N. Goncharova, O.A. Kabov, Computational Thermal Sci. 3 (4) (2011)

13. O.N. Goncharova, Izvestia AltGU 73 (1/2) (2012) 